\title{
Apolipoprotein E polymorphism interacts with cigarette smoking in progression of multiple sclerosis
}

\author{
A. Sena ${ }^{a, b, c}$, R. Couderc ${ }^{d}$, V. Ferret-Sena ${ }^{c}$, R. Pedrosa ${ }^{b}$, M. L. Andrade ${ }^{a}$, C. Araujo ${ }^{b}$, R. Roque ${ }^{b}$ \\ M. J. Cascais ${ }^{a}$ and M. G. Morais ${ }^{a}$ \\ ${ }^{a}$ Department of Biochemistry, Faculty of Medical Sciences, UNL, Lisbon, Portugal; ${ }^{b}$ Department of Neurosciences, Neurology Service, \\ Centro Hospitalar de Lisboa Central, Lisbon, Portugal; ${ }^{\mathrm{c}}$ Department of Physiology, Cooperativa Egas Moniz, Monte de Caparica, Portugal; \\ and ${ }^{\mathrm{d}}$ Laboratory of Biochemistry, Hôpital Trousseau AP-HP, Paris, France
}

\section{Keywords:}

apolipoprotein E, cigarette smoking, multiple sclerosis

Received 13 December 2008 Accepted 19 February 2009
Background and purpose: The influence of apolipoprotein E (ApoE) polymorphism on clinical severity of multiple sclerosis (MS) is still controversial. Cigarette smoking has been suggested to influence the progression of disability in these patients. In this study, we aimed to investigate whether an interaction of smoking with the ApoE polymorphism influences the progression of disability in MS patients.

Methods: Smoking history from 205 female patients with MS was obtained. Clinical data collected include age at onset, disease duration, annual relapse rate, the Expanded Disability Status Scale (EDSS) and the Multiple Sclerosis Severity Score (MSSS). ApoE polymorphism was examined in all patients and stratified according to smoking status and associations with the clinical data investigated.

Results: There were no significant associations between cigarette smoking and any of the clinical characteristics in the whole group of patients. In women carrying the ApoE E4 isoform, smokers had a lower EDSS $(P=0.033)$ and MSSS $(P=0.023)$ in comparison with non-smokers.

Conclusion: Our data suggest that in women with MS carrying the ApoE E4 isoform, cigarette smoking may have a protective influence on disease progression and accumulation of disability. These findings need to be confirmed by future large longitudinal studies.

\section{Introduction}

Evidence on the influence of apolipoprotein E (ApoE) polymorphism on clinical severity of multiple sclerosis (MS) has been controversial $[1,2]$. The ApoE gene exists in three common allelic forms corresponding to the protein isoforms E2, E3 and E4, with distinct biochemical and functional properties. ApoE has been suggested to have a role in remodelling and repairing nervous tissue and in modifying systemic and brain inflammatory responses $[3,4]$. Some studies have indeed found an association between ApoE E4 allele and an increased brain damage in these patients (for a review, see Pinholt et al. [2]). Several confounders and methodological issues could explain the discrepancies between results. In particular, it is possible that environmental and genetic factors could interact with

Correspondence: Armando Sena, Departamento de Bioquímica, Faculdade de Ciências Medicas, Universidade Nova de Lisboa, Campo Mártires da Pátria, 130, 1169-056 Lisbon, Portugal (tel.: 351 218803033/82; fax: 351 218803010; e-mail: arsena@fcm.unl.pt).
ApoE in modulating disease course [1]. One of these potential confounders is exposure to tobacco smoking.

Cigarette smoking [5,6] and smoker exposure [7] have been found to increase the risk for MS. However, an influence of smoking on disease progression and accumulation of disability $[8,9]$ was not supported by a large prospective study [10]. Interestingly, the risk associated with the ApoE polymorphism has been shown to be modulated by smoking in several situations, including cardiovascular disease [11], age-related macular degeneration [12] and Alzheimer's disease [13]. Based on these data, we hypothesized that cigarette smoking could interact with the ApoE polymorphism to modulate disease course in MS patients.

\section{Methods}

\section{Patients and smoking history}

A total of 205 female patients with MS according to the revised Mc Donald criteria [14] were recruited from the MS outpatient clinic at the Neurology Service of the Centro Hospitalar de Lisboa-Central. Our MS 
database contains laboratory and clinical data, including information on smoking habits. The smoking status, if not documented completely, was obtained by telephone information with the respective patient. In this study, we included patients aged 15-62 years and with at least 1 year of disease duration, examined between 1995 and 2007. From this database, we collected clinical information before the patients received immunomodulatory or immunosuppressive treatments. In addition, none has been treated with steroids for at least 1 month. The clinical data collected were age at onset of MS, disease duration, annual relapse rate, and disease disability and severity, according to the Expanded Disability Status Scale (EDSS) and the Multiple Sclerosis Severity Score (MSSS). The EDSS is the most validated and widely accepted measure of MS disability [15]. The MSSS is an algorithm which relates scores on the EDSS to the distribution of disability in patients with comparable disease durations. We determined the Global MSSS as detailed in the article by Roxburgh et al. [16]. The course of MS was described as relapsing-remitting $(n=163)$, secondary progressive $(n=22)$ and primary progressive $(n=$ 20). Patients gave a written consent to participate in the study, approved by the local ethics committee. Male patients were not included in this series because the cohort examined for the ApoE polymorphism was not large enough for a statistical powerful analysis of the results.

The smoking questionnaire contained questions on current smoking status, starting and quitting dates and number of cigarettes smoked. With one exception, all patients who were current smokers started smoking before the onset of MS. Ten patients stopped smoking before the onset of the disease. Patients who never smoked in their lives or reported to smoke fewer than five cigarettes occasionally (not every day) were considered non-smokers. In this study, all patients labelled smokers reported to smoke at least five cigarettes per day. We decided not to further divide these subjects according to the number of cigarettes smoked because most of them reported to change their frequency of daily consumption. There were no cigar and pipe smokers.

\section{ApoE typing}

ApoE polymorphism was examined by using an isoelectric focusing (IEF) method, as described previously [17]. Briefly, $15 \mu \mathrm{l}$ of delipidated plasma samples was run in duplicate on agarose with sorbitol, urea, ampholine $(\mathrm{pH}$ 5-7) and pharmalyte (pH 4-6.5) (Pharmacia Biotech; Amersham Pharmacia Biotech, Little Chalfont, UK). After IEF the proteins were electro transferred to nitrocellulose membranes (Immobilon, pore size $0.2 \mu \mathrm{m}$; Millipore; Millipore Corporate Headquarters, Billerica, MA, USA). The membranes were sequentially incubated with polyclonal-goat anti-human ApoE antibody (Daichi; Daichi Pure Chemicals, Tokyo, Japan) and IgG peroxidase-conjugate anti-goat antibody (Sigma; SigmaAldrich Biotechnology LP, St. Louis, MO, USA). The ApoE isoforms were visualized in a substrate solution containing 3,3'diaminobenzidine tetrahydrochloride reagent (Sigma). It has been shown that for common ApoE polymorphism, protein phenotyping is in good agreement with DNA-based genotyping [18]. ApoE typing was performed in blinded conditions regarding the clinical data and smoking history of the patients.

\section{Statistical analysis}

Age, age at onset, disease duration, annual relapse rate, EDSS and MSSS, and age at start of smoking and number of years of smoking were compared between $\mathrm{E} 3 / 3$ and $\mathrm{E} 4 / 3$ and between $\mathrm{E} 3 / 3$ and $\mathrm{E} 2 / 3$ patients, and between smoker and non-smoker patients in the ApoE phenotype subsets of subjects with analysis of variance and Bonferroni/Dunn test. The proportions of relapsing-remitting, secondary progressive and primary progressive forms were compared in the ApoE phenotype subsets of subjects with the chi-squared test. Significance was taken to be at the two-tailed 0.05 level. All statistical analysis was performed with the StatView 5.0 software.

\section{Results}

The demographic and clinical variables in the different ApoE subgroups for all women investigated are compared in Table 1. The ApoE phenotypes were E3/E3 (76.6\%), E4/E3 (12.7\%) and E2/E3 (9.3\%). Three additional patients with the E2/E4 phenotype were excluded from the analysis. No homozygotes for the E2 and E4 alleles were detected. The observed frequencies of ApoE alleles were comparable with the general populations from Portugal and other countries in South Europe [19]. No clinical differences were found to be associated with particular ApoE alleles. Start of smoking and years of smoking were also comparable in the three genetic subgroups.

Patients were then stratified according to smoking status (Table 2). No significant clinical differences between smokers and non-smokers were observed in the whole group of patients and in those with the E3/E3 and E2/E3 phenotypes. Ten patients who stopped smoking before disease onset had an E3/E3 phenotype. Excluding those subjects from the analysis did not 
Table 1 Subjects demographic and clinical characteristics according to ApoE polymorphism subgroups

\begin{tabular}{|c|c|c|c|c|c|c|}
\hline & $\begin{array}{l}\text { Total } \\
(n=202)\end{array}$ & $\begin{array}{l}\mathrm{E} 3 / \mathrm{E} 3 \\
(n=157)\end{array}$ & $\begin{array}{l}\mathrm{E} 3 / \mathrm{E} 4 \\
(n=26)\end{array}$ & $\begin{array}{l}\text { E4 versus E3 } \\
(P \text {-value })\end{array}$ & $\begin{array}{l}\mathrm{E} 2 / \mathrm{E} 3 \\
(n=19)\end{array}$ & $\begin{array}{l}\text { E2 versus E3 } \\
(P \text {-value })\end{array}$ \\
\hline Age, years & $38.69 \pm 10.35$ & $38.98 \pm 10.26$ & $38.42 \pm 10.9$ & 0.802 & $36.68 \pm 10.65$ & 0.363 \\
\hline Age at onset, years & $31.95 \pm 9.59$ & $32.08 \pm 9.41$ & $32.12 \pm 10.11$ & 0.990 & $30.63 \pm 9.92$ & 0.528 \\
\hline $\begin{array}{l}\text { Disease duration, } \\
\text { years }^{\mathrm{a}}\end{array}$ & $5.0(2.0-10.0)$ & $5.0(2.0-10.0)$ & $5.0(2.0-9.0)$ & 0.927 & $3.0(2.0-8.25)$ & 0.368 \\
\hline Annual relapse rate & $0.87 \pm 0.62$ & $0.88 \pm 0.64$ & $0.88 \pm 0.59$ & 0.980 & $0.79 \pm 0.54$ & 0.547 \\
\hline EDSS & $2.57 \pm 1.57$ & $2.61 \pm 1.61$ & $2.75 \pm 1.47$ & 0.666 & $2.08 \pm 1.35$ & 0.164 \\
\hline MSSS & $4.24 \pm 2.49$ & $4.24 \pm 2.50$ & $4.59 \pm 2.19$ & 0.530 & $3.77 \pm 2.85$ & 0.423 \\
\hline \multicolumn{7}{|l|}{ Disease course, $n(\%)$} \\
\hline RRMS & $161(79.7)$ & $122(77.7)$ & $21(80.7)$ & & $18(94.7)$ & \\
\hline SPMS & $22(11.1)$ & $19(12.1)$ & $3(11.5)$ & 0.916 & 0 & 0.187 \\
\hline PPMS & $19(9.4)$ & $16(10.2)$ & $2(7.7)$ & & $1(5.2)$ & \\
\hline Smokers, $n(\%)$ & $72(35.6)$ & $51(32.5)$ & $11(42.3)$ & & $10(52.6)$ & \\
\hline $\begin{array}{l}\text { Start of smoking, } \\
\text { years }\end{array}$ & $17.29 \pm 3.74$ & $17.25 \pm 3.83$ & $16.64 \pm 2.25$ & 0.623 & $18.2 \pm 4.71$ & 0.471 \\
\hline Years of smoking & $20.93 \pm 8.76$ & $21.78 \pm 9.49$ & $19.91 \pm 5.84$ & 0.521 & $17.7 \pm 7.07$ & 0.182 \\
\hline
\end{tabular}

Unless otherwise indicated, data are expressed as mean \pm SD. Groups compared by analysis of variance and Bonferroni/Dunn test. The proportions of disease forms were compared by chi-squared test. EDSS, Expanded Disability Status Scale; MSSS, Multiple Sclerosis Severity

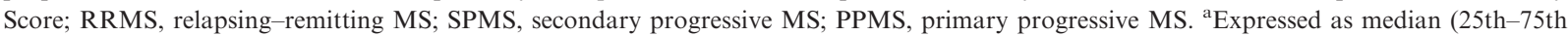
percentile); $P$ calculated as log-transformed data.

Table 2 Clinical characteristics in ApoE allele subgroups stratified according to smoking status

\begin{tabular}{|c|c|c|c|c|c|c|}
\hline & Age, years & $\begin{array}{l}\text { Age at onset, } \\
\text { years }\end{array}$ & $\begin{array}{l}\text { Disease duration, } \\
\text { years }^{\mathrm{a}}\end{array}$ & $\begin{array}{l}\text { Annual } \\
\text { relapse rate }\end{array}$ & EDSS & MSSS \\
\hline \multicolumn{7}{|l|}{ Total } \\
\hline NS (130) & $38.95 \pm 11.31$ & $31.74 \pm 10.08$ & $5.0(2.0-10.0)$ & $0.84 \pm 0.68$ & $2.72 \pm 1.60$ & $4.38 \pm 2.51$ \\
\hline SM (72) & $38.2 \pm 8.5$ & $32.35 \pm 8.76$ & $4.0(2.0-8.5)$ & $0.92 \pm 0.50$ & $2.31 \pm 1.50$ & $4.03 \pm 2.44$ \\
\hline$P$ value & 0.636 & 0.666 & 0.274 & 0.390 & 0.075 & 0.335 \\
\hline \multicolumn{7}{|l|}{ E3/E3 } \\
\hline NS (106) & $38.94 \pm 10.93$ & $31.47 \pm 9.93$ & $5(2.0-10.0)$ & $0.85 \pm 0.69$ & $2.69 \pm 1.57$ & $4.27 \pm 2.49$ \\
\hline $\mathrm{SM}(51)$ & $39.04 \pm 8.96$ & $33.37 \pm 8.29$ & $3.0(2.0-7.75)$ & $0.95 \pm 0.52$ & $2.46 \pm 1.67$ & $4.23 \pm 2.52$ \\
\hline$P$ value & 0.956 & 0.238 & 0.180 & 0.344 & 0.403 & 0.917 \\
\hline \multicolumn{7}{|l|}{ E4/E3 } \\
\hline NS (15) & $39.8 \pm 13$ & $34.07 \pm 11.43$ & $5.0(2.25-5.75)$ & $0.87 \pm 0.69$ & $3.27 \pm 1.68$ & $5.40 \pm 2.22$ \\
\hline SM (11) & $36.5 \pm 7.35$ & $29.45 \pm 9.37$ & $6.0(2.0-11.75)$ & $0.91 \pm 0.44$ & $2.05 \pm 0.72$ & $3.47 \pm 1.66$ \\
\hline$P$ value & 0.463 & 0.284 & 0.830 & 0.860 & 0.033 & 0.023 \\
\hline \multicolumn{7}{|l|}{$\mathrm{E} 2 / \mathrm{E} 3$} \\
\hline NS (9) & $37.56 \pm 13.93$ & $31.0 \pm 10.17$ & $3.0(1.0-10.25)$ & $0.78 \pm 0.62$ & $2.33 \pm 1.66$ & $3.94 \pm 3.05$ \\
\hline SM (10) & $35.9 \pm 7.28$ & $30.3 \pm 10.22$ & $3.5(2.0-6.0)$ & $0.80 \pm 0.48$ & $1.85 \pm 1.03$ & $3.61 \pm 2.81$ \\
\hline$P$ value & 0.745 & 0.883 & 0.794 & 0.931 & 0.450 & 0.808 \\
\hline
\end{tabular}

Unless otherwise indicated, data are expressed as mean \pm SD. NS (non-smoking) versus SM (smoking) groups compared by analysis of variance and Bonferroni/Dunn test. EDSS, Expanded Disability Status Scale; MSSS, Multiple Sclerosis Severity Score. ${ }^{a}$ Expressed as median (25th-75th percentile); $P$ calculated as log-transformed data.

change the results. In patients carrying the $\mathrm{E} 4$ isoform, smokers had a lower EDSS $(P=0.033)$ and a lower $\operatorname{MSSS}(P=0.023)$ in comparison with non-smokers. In these subjects, as shown in Fig. 1, half of the nonsmokers had an EDSS value and an MSSS value above 3.5 and 6, respectively, and none of the smoker subjects was above these thresholds. Analysis of covariance (ANCOVA) was performed in order to take into account a possible covariation of the dependent variable (EDSS or MSSS) with age and disease duration or with age and age at disease onset in apoE 3/4 patients. Irrespective of the combination of covariables, EDSS and MSSS were significantly lower in smokers than in non-smokers. When the analysis was restricted to relapsing-remitting cases these differences became nonsignificant, possibly due to fewer numbers of subjects included.

\section{Discussion}

Our cross-sectional study included one of the largest cohorts of women with MS assessed for evaluating the 


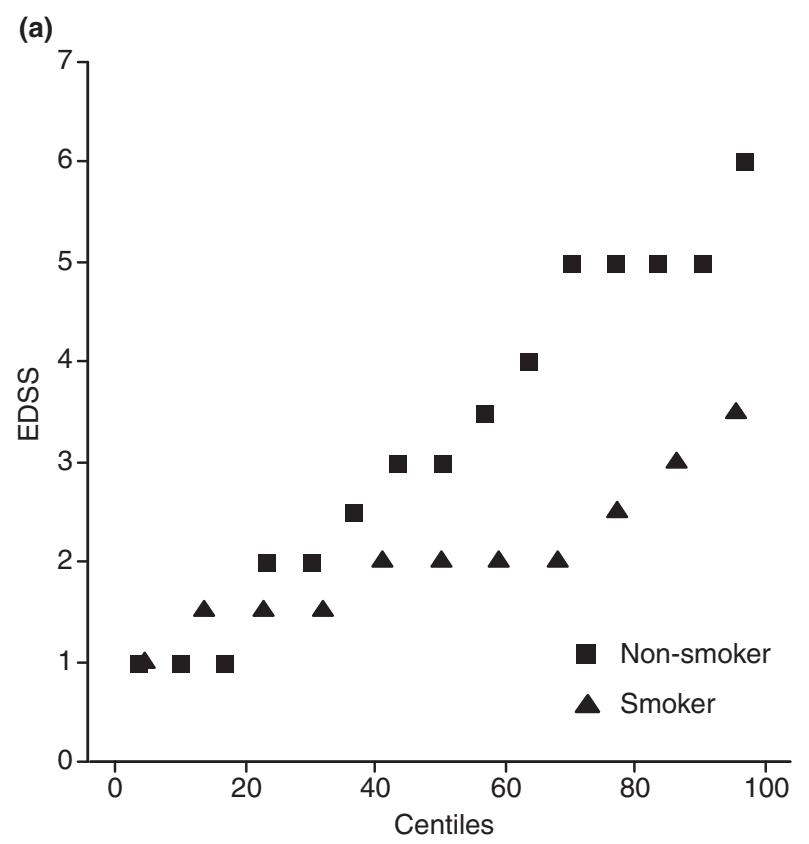

(b)

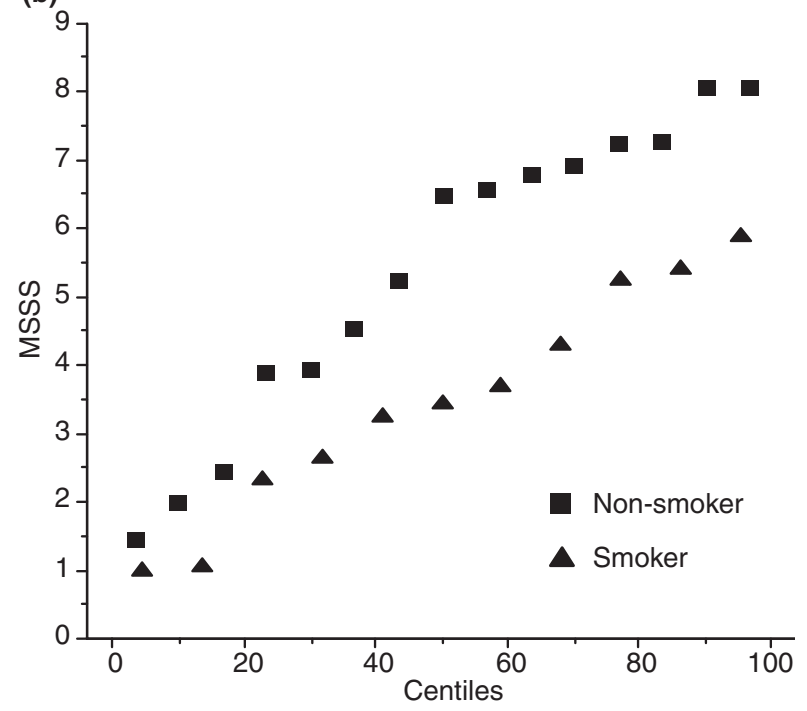

Figure 1 Expanded Disability Status Scale (EDSS; a) and Multiple Sclerosis Severity Score (MSSS; b) percentile values in non-smoker (squares) and smoker (triangles) patients carrying the APOE E4 allele.

influence of ApoE polymorphism on disease severity. In agreement with a previous study [1] our study did not find any clinical differences associated with ApoE alleles in the whole group of patients. Studies in patients with a similar short duration of the disease $(<5$ years) have shown a deleterious effect of the apoE4 allele on brain structural integrity [2]. However, the potential pathogenic impact of this isoform suggested by this work was not reflected in significant effects on clinical disability.
Although previous studies on the influence of the ApoE E4 allele on disability progression of MS have been contradictory, none included information about smoking history. Our data indicate that smoking status may influence the impact of this allele on clinical progression in the earliest phase of the disease. It is now well established that neuroaxonal damage begins in the earliest stages of MS and greatly contributes to the progression of disability late in the course of the disease [20]. The global MSSS is considered to be the most powerful of the current methods for measurement severity of MS $[1,16]$ and was suggested to correlate with axonal damage in these patients [21]. Therefore, a lower progression rate (MSSS) and disability (EDSS) in smokers in comparison with non-smokers in patients carrying the E4 isoform suggests a neuroprotective effect associated with cigarette smoking. However, it should be noted that in agreement with another work [10], no associations were found between smoking status and the clinical measures in the whole study population. This is in contrast to two other studies [8,9], suggesting an association between ever-smoking and progressive disease. Differences in methodology, characteristics of patients and sample size may account for this discrepancy. An analysis of these studies suggests that differences in age at the start of smoking, the daily amount of smoked cigarettes and the smoking status before versus after the onset of MS, may contribute to conflicting results. Moreover, as discussed in a recent paper [7], there are individual and gender-related differences in the metabolism of smoked substances that could variably interfere with mechanisms associated with MS. Finally, the confounding of immunomodulatory treatments or other environmental factors and the interaction of smoking with genetic factors implicated in immune response, such as the HLA-DR haplotype [7], should also be considered.

Although many tobacco smoke compounds or smoke-related metabolites have toxic effects on the central nervous system, nicotine possibly promotes protective mechanisms against certain inflammatory and neurodegenerative processes [13,22,23]. Nicotine has systemic immunosuppressive effects [22], inhibits microglial activation [24] and leucocyte recruitment during inflammation [25] and has anti-excitotoxic [26] and antioxidative [27] properties. These effects are mainly mediated through $\alpha 7$ nicotinic cholinergic receptors. ApoE E4 isoform, in comparison with the other isoforms, is suggested to be linked to greater systemic and brain inflammatory reaction and impairment of neural repair [3,4]. We could speculate that in MS patients, smoking could counterbalance the ApoE E4-associated promotion of brain tissue damage by facilitating nicotinic cholinergic function. An impair- 
ment of brain cholinergic pathways has been shown to occur in experimental allergic encephalomyelitis (EAE) [28] and in MS patients [29,30]. Moreover, the acetylcholinesterase inhibitor rivastigmine was recently observed to reduce neuroinflammmatory and axonal damage in EAE through $\alpha 7$ nicotinic receptors [31]. Taken together, our results and the reviewed data could suggest an involvement of nicotinic receptors in the pathogenesis of MS and that selective nicotinic pharmacological agents should be explored as potential useful neuroprotective strategies for these patients.

In conclusion, our results suggest that in MS patients carrying the apoE4 isoform, cigarette smoking may have a protective influence on disability progression. We recognize that this study has limitations owing to the cross-sectional design and restriction to a population of female patients. In order to confirm our data further large longitudinal studies will be required. Nevertheless, our findings may help explain, at least in part, the discrepancies between individual studies investigating separately the influence of $\mathrm{ApoE}$ and smoking in disease severity of MS.

\section{Acknowledgements}

We thank MS nurses Cristina Araujo and Ana Mendes for help in research and kind assistance to our patients. This work was in part supported by grants from Bayer Schering Pharma, Merck Serono and Sanofi-aventis.

\section{References}

1. Burwick RM, Ramsay PP, Haines JL, et al. APOE epsilon variation in multiple sclerosis susceptibility and disease severity. Some answers. Neurology 2006; 66: 13731383.

2. Pinholt M, Frederiksen JL, Christiansen M. The association between apolipoprotein $\mathrm{E}$ and multiple sclerosis. Eur J Neurol 2006; 13: 573-580.

3. Lynch JR, Tang W, Wang H, et al. ApoE genotype and ApoE-mimetic peptide modify the systemic and central nervous system inflammatory response. J Biol Chem 2003; 278: 48529-48533.

4. Mahley RW, Weisgraber KH, Huang Y. Apolipoprotein E4: causative factor and therapeutic target in neuropathology, including Alzheimer's disease. Proc Natl Acad Sci USA 2006; 103: 5644-5651.

5. Hawkes $\mathrm{CH}$. Smoking is a risk factor for multiple sclerosis: a metanalysis. Mult Scler 2007; 13: 610-615.

6. Di Pauli F, Reindl M, Ehling R, et al. Smoking is a risk factor for early conversion to clinically definite multiple sclerosis. Mult Scler 2008; 14: 1026-1030.

7. Sundström P, Nyström L, Hallmans G. Smoke exposure increases the risk for multiple sclerosis. Eur J Neurol 2008; 15: 579-583.

8. Hernán MA, Jick SS, Logroscino G, Olek MJ, Ascherio A, Jick H. Cigarette smoking and progression of multiple sclerosis. Brain 2005; 128: 1461-1465.
9. Sundström P, Nyström L. Smoking worsens the prognosis in multiple sclerosis. Mult Scler 2008; 14: 1031-1035.

10. Koch M, van Harten A, Uyttenboogaart M, De Keyser J. Cigarette smoking and progression in multiple sclerosis. Neurology 2007; 69: 1515-1520.

11. Talmud PJ, Stephens JW, Hawe E, et al. The significant increase in cardiovascular disease risk in ApoE epsilon4 carriers is evident only in men who smoke: potential relationship between reduced antioxidant status and ApoE4. Ann Hum Genet 2005; 69: 613-622.

12. Schmidt S, Haines JL, Postel EA, et al. Joint effects of smoking history and APOE genotypes in age-related macular degeneration. Molecular Vision 2005; 11: 941-949.

13. Reitz C, den Heijer T, Duijn van C, Hofman A, Breteler MMB. Relation between smoking and risk of dementia and Alzheimer disease. The Rotterdam study. Neurology 2007; 69: 998-1005.

14. Polman $\mathrm{CH}$, Reingold SC, Edan G, et al. Diagnostic criteria for multiple sclerosis: 2005 Revisions to the "Mc Donald Criteria". Ann Neurol 2005; 58: 840-846.

15. Kurtzke JF. Rating neurologic impairment in multiple sclerosis: and Expanded Disability Status Scale (EDSS). Neurology 1983; 33: 1444-1452.

16. Roxburgh RHSR, Seaman SR, Masterman T, et al. Multiple sclerosis severity score. Using disability and disease duration to rate disease severity. Neurology 2005; 64: 1144-1151.

17. Bailleul S, Couderc R, Landais G, Lefevre D, Raichvarg D, Etienne J. Direct phenotyping of human apolipoprotein $\mathrm{E}$ in plasma: application to population frequency distribution in Paris (France). Hum Hered 1993; 43: 159165.

18. Hansen PS, Gerdes LU, Klausen IC, Gregersen N, Faergeman $\mathrm{O}$. Genotyping compared with protein phenotyping of the common apolipoprotein E polymorphism. Clin Chim Acta 1994; 224: 131-137.

19. Santos M, Costa MC, Rio ME, et al. Genotypes of the APOE and SCA2 loci do not predict the course of multiple sclerosis in patients of Portuguese origin. Mult Scler 2004; 10: 153-157.

20. Trapp BD, Nave KA. Multiple sclerosis: an immune or neurodegenerative disorder? Annu Rev Neurosci 2008; 31: 247-269.

21. Petzold A, Eikelenboom MJ, Polman $\mathrm{CH}$, et al. The new Global Multiple Sclerosis Severity Score (MSSS) correlates with axonal but not glial biomarkers. Mult Scler 2006; 12: 325-328.

22. Sopori M. Effects of cigarette smoke on the immune system. Nat Rev Immunol 2002; 2: 372-377.

23. Louis ED, Benito-Léon J, Bermejo-Pareja F. Populationbased prospective study of cigarette smoking and risk of incident essential tremor. Neurology 2008; 70: 1682-1687.

24. Shytle RD, Mori T, Townsend K, et al. Cholinergic modulation of microglial activation by alpha 7 nicotinic receptors. J Neurochem 2004; 89: 337-343.

25. Saeed RW, Varma S, Peng-Nemeroff T, et al. Cholinergic stimulation blocks endothelial cell activation and leukocyte recruitment during inflammation. J Exp Med 2005; 201: $1113-1123$.

26. Gahring LC, Meyer EL, Rogers SW. Nicotine-induced neuroprotection against $\mathrm{N}$-methyl-D-aspartic acid or beta-amyloid peptide occur through independent mechanisms distinguished by pro-inflammatory cytokines. J Neurochem 2003; 87: 1125-1136. 
27. Egea J, Rosa AO, Cuadrado A, Garcia AG, López MG. Nicotinic receptor activation by epibatidine induces heme oxigenase- 1 and protects chromaffin cells against oxidative stress. J Neurochem 2007; 102: 1842-1852.

28. D'Intino G, Paradisi M, Fernandez M, et al. Cognitive deficit associated with cholinergic and nerve growth factor down-regulation in experimental allergic encephalomyelitis in rats. Proc Natl Acad Sci USA 2005; 102: 3070-3075.

29. Krupp LB, Christodoulov C, Melville P, Scheil WF, Mac Allister WS, Elkins LE. Donepezil improves memory in multiple sclerosis in a randomized clinical trial. Neurology 2004; 63: 1579-1585.

30. Darvesh S, Reid GA, LeBlanc A, Bhan V, Fisk FD, Macaulay RJ. Butyrylcholinesterase activity in multiple sclerosis. Mult Scler 2008; 14(Suppl. 1): 821. (Abstract)

31. Nizri E, Irony-Tur-Sinai M, Faranesh N, et al. Suppression of neuroinflammation and immunomodulation by the acetylcholinesterase inhibitor rivastigmine. $J$ Neuroimmunol 2008; 203: 12-22. 
Copyright of European Journal of Neurology is the property of Blackwell Publishing Limited and its content may not be copied or emailed to multiple sites or posted to a listserv without the copyright holder's express written permission. However, users may print, download, or email articles for individual use. 